\title{
Primera evidencia experimental mediante microscopía electrónica de transmisión de la disociación de dislocaciones en el sistema $\mathrm{TeO}_{2}$
}

\author{
Á. GALLARDO-LÓPEZ, D. GÓMEZ-GARCÍA, A. DOMÍNGUEZ-RODRíGUEZ , J. DOUIN Y J. CASTAING \\ Departamento de Física de la Materia Condensada-Universidad de Sevilla \\ Apartado 1065, 4180 Sevilla, España \\ + Centre d'Elaboration de Matériaux et d'Etudes Structurals" (CEMES) \\ 29, rue Jeanne Marig, BP 94347. 31,55 Toulouse Cedex 4, France \\ Centre de Recherche et de Restauration des Musées de France \\ C2RMF -- LRMF - C.N.R.S. UMR 171 \\ Palais du Louvre - Porte des Lions, 14 quai François Mitterand 75001 Paris, France
}

\begin{abstract}
En este trabajo se pretende analizar la microestructura de monocristales de $\mathrm{TeO}_{2}$ (paratelurita) deformados plásticamente en compresión uniaxial a alta temperatura $(\sim 870 \mathrm{~K})$ en distintas condiciones. Para ello, se han ensayado muestras con orientación cristalográfica [110], y se han cortado láminas correspondientes a los planos de deslizamiento para ser observadas con microscopía electrónica de transmisión. El estudio de la subestructura de dislocaciones en este material es complejo debido a su alta anisotropía. La observación de dicha microestructura ha puesto de manifiesto la presencia de disociación de dislocaciones. Es la primera vez que se encuentra este fenómeno en la paratelurita. De la distancia de equilibrio entre dislocaciones parciales, se ha podido obtener una estimación de la energía de falta de apilamiento en este sistema.
\end{abstract}

Palabras clave: $\mathrm{TeO}_{2}$, paratelurita, monocristal, dislocaciones, $\mathrm{MET}$

\section{A first experimental evidence by transmission electron microscopy of dislocation disociation in the $\mathrm{TeO}_{2}$ system}

This work intends to analyse the microstructure of paratellurite $\left(\mathrm{TeO}_{2}\right.$ single crystals) plastically deformed at high temperature $(\sim 870 \mathrm{~K})$ in uniaxial compression along [110]. Samples have been mechanically tested, and thin foils have been cut from them, corresponding to the slip systems to be observed in transmission electron microscopy. The study of the dislocation substructure in this material is rather complex due to its high anisotropy. Microstructural observation by TEM has put into evidence the presence of dislocation dissociation. This is the first work reporting this fact. From the equilibrium distance between partials, an estimation of the stacking fault energy has been carried out.

Keywords: $\mathrm{TeO}_{2}$, paratellurite, single crystal, dislocations, TEM.

\section{INTRODUCCIÓN}

Los cristales de dióxido de teluro $\left(\mathrm{TeO}_{2}\right)$ se emplean en la fabricación de dispositivos acusto-ópticos. La luz visible (longitud de onda entre 0.33 y $4 \mu \mathrm{m}$ ) puede ser difractada por una onda ultrasonora, permitiendo así fabricar moduladores, deflectores, analizadores espectrales, filtros sintonizables, etc. $(1,2)$. Aunque el empleo de este material está en constante crecimiento, no existen datos suficientes sobre imperfecciones cristalinas tales como las dislocaciones, que afectan notablemente a la atenuación de las ondas acústicas (3) y, por consiguiente, a la calidad de los dispositivos. Esta influencia está relacionada con las tensiones internas que rodean a las dislocaciones. En este sentido, $\mathrm{el} \mathrm{TeO}_{2}$ es un caso de estudio muy interesante. Un trabajo reciente sobre las aplicaciones de este material en dispositivos acusto-ópticos se encuentra en (4). Este material presenta una transición de fase ferroelástica a temperatura ambiente y presión hidrostática de $900 \mathrm{MPa}$ aproximadamente. El cálculo del campo de tensiones alrededor de las dislocaciones podría predecir si se produce alguna transición de fase en las proximidades de las líneas de dislocación, ya que esto afectaría a su movimiento. Posee una estructura tetragonal $(\mathrm{a}=0.48 \mathrm{~nm} ; \mathrm{c}=0.76 \mathrm{~nm})$, y puede derivarse de la estructura rutilo si se superponen dos celdas unidad a lo largo de la dirección [001], pero es un cristal fuertemente anisótropo desde el punto de vista elástico. Considerando todas las direcciones cristalográficas, la razón entre los módulos de Young máximo y mínimo es de 11.3 $(5,6)$, mientras que para otros cristales anisótropos, como la calcita y el cuarzo, no sobrepasa los valores de 2.9 y 1.9 respectivamente (5). Esta situación tan peculiar estimuló un estudio preliminar de dislocaciones, atendiendo a los aspectos macroscópicos de la deformación plástica y a la determinación de sistemas de deslizamiento a partir de la energía elástica de las dislocaciones (5), que no ha tenido continuidad. En dicho trabajo, se deformaron plásticamente monocristales de $\mathrm{TeO}_{2}$ para determinar los sistemas de deslizamiento activos y compararlos con las predicciones teóricas. Los cristales son frágiles a temperatura ambiente cuando se les somete a compresión, y la ductilidad sólo aparece a partir de los $670 \mathrm{~K}$. La deformación tuvo lugar en compresión uniaxial a lo largo de distintas direcciones cristalográficas, y a una temperatura lo suficientemente alta $\left(\mathrm{T}>0.7 \mathrm{~T}_{\mathrm{f}}\right.$, donde $\mathrm{T}_{\mathrm{f}}$ es la temperatura de fusión, $1010 \mathrm{~K}$ ). Durante la deformación plástica en compresión se activan varios sistemas de deslizamiento. Combinando los cálculos de las energías de las dislocaciones 
y las observaciones macroscópicas de los cristales deformados mediante topografía de rayos X Berg-Barret, se concluyó que los planos de deslizamiento activados eran el $\{100\},(001),\{110\}$ y $\{101\}$. También se sugirió la existencia de vectores de Burgers $<010>$ y $<110>$.

Posteriormente, Yasutake et al (7) llevaron a cabo un primer estudio de microscopía electrónica de transmisión de muestras deformadas. Se confirmó la activación de los sistemas activación de deslizamiento $<100>(001),<1 \overline{\mathbf{I}} 0>\{110\}$ y $<100>\{010\},<1 \overline{\mathbf{T}} 0>\{110\}$ en acuerdo con (5). Además, propusieron la existencia del sistema $<110>(001)$, excluido por (5) de los sistemas activos. En este último sistema, existe una diferencia muy notable entre las energías elásticas de las dislocaciones en arista y helicoidales, debido a la alta anisotropía del $\mathrm{TeO}_{2}$. Estos autores concluyeron además que es difícil predecir cuál es el sistema de deslizamiento más fácil a partir de estimaciones de la tensión de Peierls, debido a la compleja estructura cristalina y a la anisotropía del $\mathrm{TeO}_{2}$. Según (8) la tensión de Peierls se obtiene mediante la derivación de la energía máxima de la dislocación por unidad de longitud, y puede escribirse como:

$$
\tau,=\frac{2 G}{(1-\tau)} \operatorname{miq}\left(\frac{-2 \pi w}{b}\right)
$$

donde $G$ es el módulo de cizalladura, $v$ es el módulo de Poisson, w es la anchura de la dislocación (que depende de la naturaleza de las fuerzas interatómicas) y b el vector de Burgers. Al estar relacionado con la anchura de la dislocación, es necesario estudiar la estructura del núcleo de las dislocaciones de distintos tipos para una discusión más profunda.

Este trabajo tiene por objetivo retomar ciertos aspectos del trabajo precedente, $\mathrm{y}$, sobre todo, proceder a un examen detallado de las dislocaciones utilizando la microscopía electrónica de transmisión mediante la condición de dos haces. En un futuro se espera utilizar la técnica de haz débil para la observación del núcleo de las dislocaciones.

\section{PARTE EXPERIMENTAL}

El óxido de teluro monocristalino usado fue crecido por el método de Czochralski $(2,3)$ partiendo de material en bruto de pureza 0,99999. El eje de crecimiento fue [110]. La elección de esta orientación no es simple: coincide con la explorada de forma pionera en (7) por un lado, por lo que la comparación es posible. Pero sobre todo, estos autores probaron que en esta orientación, el criterio de extinción de dislocaciones convencional puede ser usado, con lo que puede determinarse la naturaleza de éstas sin recurrir al formalismo más general y más complejo de la teoría elástica de medios anisótropos. Se cortaron paralelepípedos de dimensiones aproximadas $3,5 \times 4 \times 8,5 \mathrm{~mm}$, orientados previamente mediante la técnica Laue de difracción de rayos X. Las orientaciones de las caras laterales, el eje de compresión y los sistemas de deslizamiento activos durante la deformación están representados esquemáticamente en la figura 1 . Se pulieron las caras laterales con pasta de diamante hasta $0.3 \mu \mathrm{m}$. Las muestras fueron sometidas a ensayos de compresión uniaxial a velocidad constante, aplicándose la tensión en el eje mayor. Los ensayos se realizaron en una máquina Instron de tipo 1185.

Un ejemplo de ensayo de deformación puede verse en la

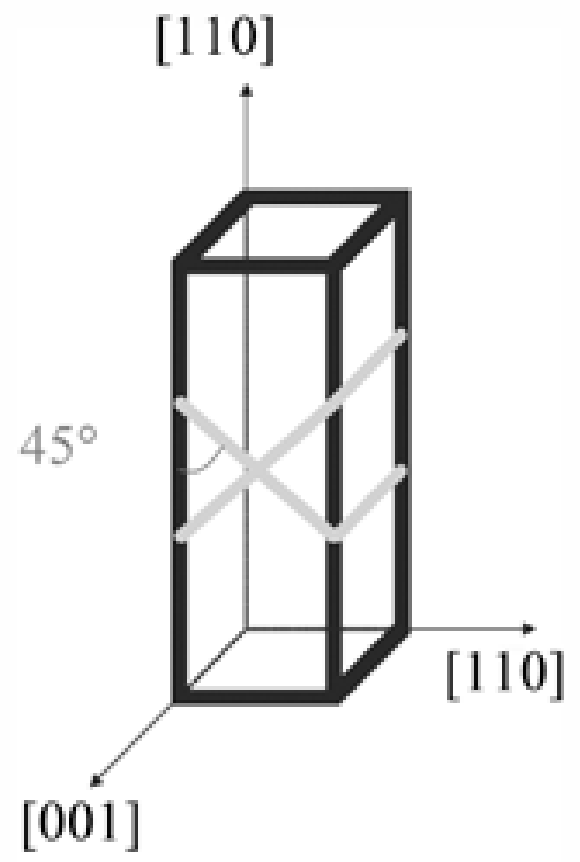

Fig. 1- Representación esquemática de las muestras cortadas para los ensayos mecánicos de compresión mostrando los sistemas de deslizamiento activados.

figura 2, que corresponde a una muestra deformada a una temperatura de $700 \mathrm{~K}$ a una velocidad de deformación de $1.5 \times 10^{-5} \mathrm{~s}^{-1}$. Hemos deformado sólo un $1 \%$, para poder hacer una buena observación de la estructura de dislocaciones, evitando que se produzca una excesiva interacción entre ellas.

La tabla I resume los posibles sistemas de deslizamiento conocidos en este material, en función de su orientación cristalográfica. De las muestras deformadas se cortaron láminas que fueron adelgazadas mecánicamente mediante pulido hasta que su espesor era próximo a unas $30 \mu \mathrm{m}$. Con posterioridad, la lámina delgada fue pegada a una anilla de cobre, y su proceso de adelgazamiento continuó mediante bombardeo iónico en adelgazador iónico hasta su transparencia electrónica. Su observación se llevó a cabo en un microscopio electrónico de transmisión Hitachi H-800 que opera a $200 \mathrm{kV}$ del Servicio de Microscopía de la Universidad de Sevilla.

TABLAI. SISTEMASDE DESLIZAMIENTO ACTIVADOS EN ENSAYOSDEDEFORMACIÓN PARA VARIAS DIRECCIONES DE COMPRESIÓN Y TENSIÓN CRÍTICA RESUELTA, SEGÚN [5].

\begin{tabular}{|c|c|c|c|}
\hline $\begin{array}{c}\text { Eje de } \\
\text { compresión }\end{array}$ & $\begin{array}{c}\text { Planos de } \\
\text { deslizamiento }\end{array}$ & $\begin{array}{c}\text { Direcciones de } \\
\text { deslizamiento }\end{array}$ & $\begin{array}{c}\text { Tensión crítica } \\
\text { resuelta (MPa) } \\
\text { (para 870 K) }\end{array}$ \\
\hline$[110]$ & $(100),(010)$ & {$[010],[100]$} & 17 \\
\hline$[221]$ & $(001)$ & {$[010],[100]$} & 4.2 \\
\hline$[100]$ & $(110),(1 \mathbf{I} 0)$ & {$[1 \mathbf{I} 0]$} & 22 \\
\hline$[001]$ & $(101),(011),($ & {$[10 \mathbf{I}],[01 \mathbf{I}]$,} & \\
\hline & $(\mathbf{I} 01),(0 \mathbf{I} 1)$ & {$[101],[011]$} & \\
\hline
\end{tabular}




\section{RESULTADOS Y DISCUSIÓN}

Las figuras $2 \mathrm{a}$ y $2 \mathrm{~b}$ muestran micrografías de transmisión del plano de deslizamiento (100) de una muestra de $\mathrm{TeO}_{2}$ deformada a $870 \mathrm{~K}$ con el eje de compresión en la dirección [110]. Las dislocaciones son largas, observándose una fuerte interacción y anclaje de las mismas. Nótese asimismo la aparición de algunos "escalones" sobre la línea de las dislocaciones. Tales escalones o kinks corresponden a valles del potencial de Peierls: en todo momento las líneas de las dislocaciones tienden a situarse en los puntos de mínima energía del potencial de Peierls (Ver detalle en figura 2(a)). La micrografía 2(b) muestra que la mayor parte de las dislocaciones "post-mortem" son de tipo "en arista", ya que su vector de Burgers en este caso es perpendicular a la línea de dislocaciones y paralelo al vector $\mathrm{g}$ de la red recíproca considerado. Este hecho está en buena concordancia con las observaciones efectuadas por (7).
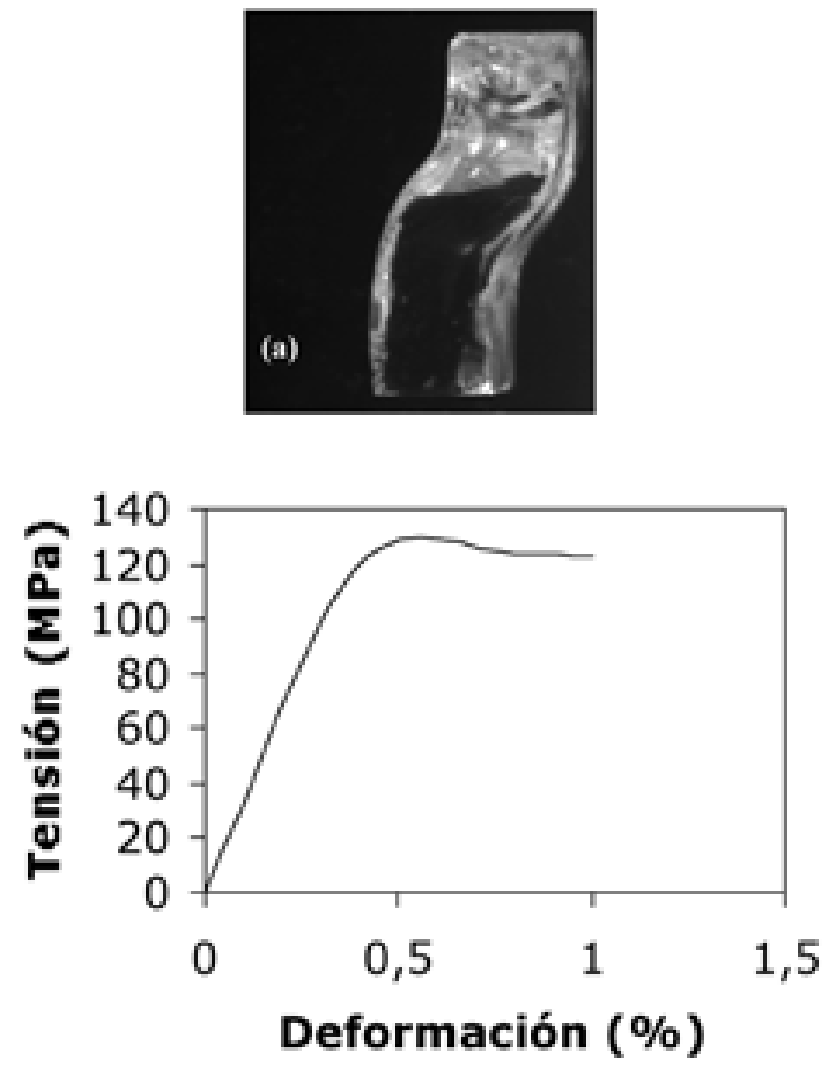

Fig. 2- Muestra deformada a $870 \mathrm{~K}$ a lo largo del eje de compresión paralelo a la dirección [110] (a) y curva tensión-deformación correspondiente (b).

Estas características son típicas de un mecanismo de Peierls, lo cual concuerda con la hipótesis propuesta por (5), que apunta a éste como mecanismo de deformación a altas temperaturas, y correlacionado con el hecho de que no puede hablarse de una energía de activación característica, independiente de la tensión aplicada.

Un hecho destacado es la presencia de contrate doble, que tiene su origen probablemente en la disociación de las dislocaciones; esto es, la aparición de dos parciales rectas separadas entre sí una distancia de alrededor de 1,5 nm
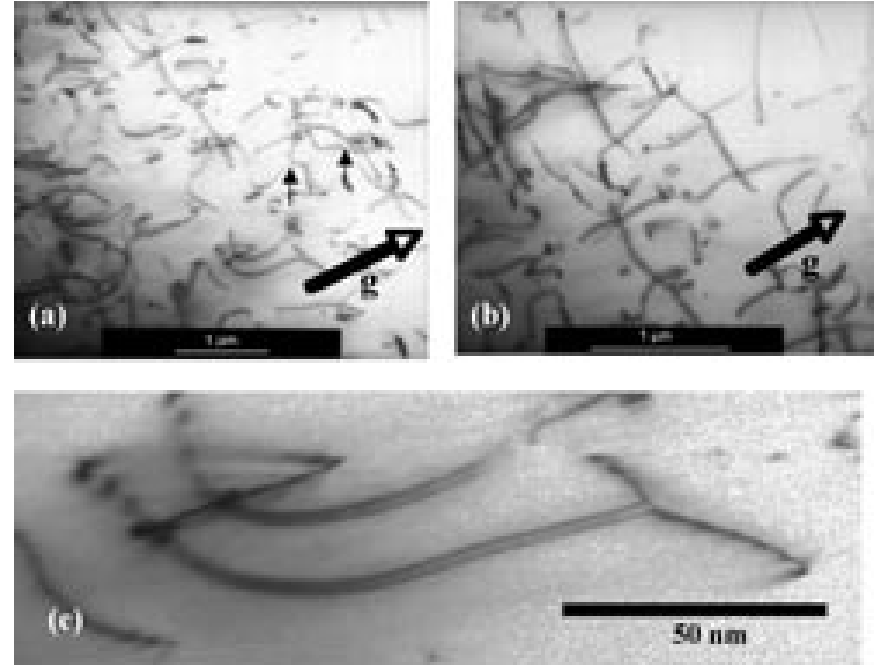

Fig. 2- Micrografías de transmisión mostrando la subestructura de dislocaciones de una muestra de $\mathrm{TeO}_{2}$ deformada a lo largo de la dirección cristalográfica [110]. En la micrografía (a) se señalan evidencias de kinks mediante flechas. Se muestra detalle de 3(c), en el que se aprecia la disociación de dislocaciones. El vector g utilizado era de tipo [020].

(ver detalle de la fig. 3). No existe hasta ahora ninguna referencia en la literatura sobre la existencia de disociación de dislocaciones en este material, pero sí existen imágenes con idéntico contraste en óxidos de estructura espinela; contraste que fue explicado mediante disociación de las dislocaciones perfectas (9). El mecanismo de esta disociación depende esencialmente de la minimización de energía que se pone en juego al escindir una dislocación en dos parciales y aparecer en su lugar una falta de apilamiento entre ambas.

Es posible hacer una estimación de la energía de falta de apilamiento puesta en juego en la disociación de una dislocación en arista en dos parciales. En la situación de equilibrio, la repulsión entre las dos dislocaciones parciales se compensa con la fuerza de atracción debida a la tendencia del sistema a reducir la falta de apilamiento. En estas condiciones, se cumple que (8):

$$
\gamma=\frac{G b^{2}}{4 \pi d}
$$

donde $\mathrm{d}$ es la distancia de equilibrio entre parciales.

Los vectores de Burgers de las dislocaciones disociadas no se conocen, pero las reacciones más plausibles pueden ser del tipo:

$$
\begin{aligned}
& {[010] \rightarrow 1 / 2[110]+1 / 2[\overline{\mathbf{I}} 10]} \\
& {[010] \rightarrow 1 / 2[010]+1 / 2[010]}
\end{aligned}
$$

La reacción [3] mantiene invariable la energía elástica en el caso de un medio elástico isótropo, mientras que la reacción [4] reduce la energía elástica de la dislocación original en un $50 \%$. Teniendo en cuenta que existe la energía de falta de apilamiento que debe sumarse a las parciales, la reacción 3 parece que no es posible. En cualquier caso, la mantenemos dada la fuerte anisotropía en este sistema, que puede inducir violaciones en la regla de Frank. Un estudio sistemático de estas parciales exige el cálculo numérico de la energía elástica 
de las dislocaciones en medios anisótropos, que es objeto de análisis en la actualidad por nuestro equipo.

Conocidos los valores numéricos para nuestro material $\left(\mathrm{G}=2.6 \times 10^{10} \mathrm{~Pa}, \mathrm{~b}=4.8 \times 10^{-10} \mathrm{~m}\right)(5,6)$, se obtiene un valor para la energía de falta de apilamiento de $\gamma \cong 80-160 \mathrm{mJm}^{-2}$, dependiendo de cuál de las dos reacciones sea la que se da de forma efectiva en este sistema. La determinación de la reacción favorable en el sistema $\mathrm{TeO}_{2}$ es objeto de estudio en la actualidad. En cualquiera de los casos, una energía de falta de apilamiento comprendida en dicho intervalo estaría asociada a una barrera de energía $\gamma \mathrm{d}^{2} \approx 1-2 \mathrm{eV}$. Esta barrera de energía es comparable con la medida en otros sistemas cerámicos, como la circona tetragonal (en el que el valor de $\gamma$ se sitúa entre 0.2 y $\left.0.4 \mathrm{Jm}^{-2}\right)(10)$, y en el que se ha invocado un mecanismo tipo Peierls para la deformación plástica a bajas temperaturas e intermedias (5). Nuestro valor es consistente pues con la existencia de un mecanismo tipo Peierls.

Un cálculo teórico más detallado exige el conocimiento de la naturaleza cristalográfica de dicha falta de apilamiento, la cual será objeto de estudio en posteriores trabajos mediante Microscopía Electrónica de haz débil.

\section{CONCLUSIONES}

Este trabajo es un estudio preliminar de la microestructura de las dislocaciones originadas en la deformación plástica de monocristales de $\mathrm{TeO}_{2}$. El estudio ha permitido poner de manifiesto que un mecanismo de Peierls es consistente con los datos mecánicos existentes en la literatura. Un hecho muy relevante es la presencia de dislocaciones disociadas, dando lugar al correspondiente par de parciales. La naturaleza cristalográfica de la falta de apilamiento será objeto de estudio en trabajos posteriores, fundamentalmente mediante microscopía de haz débil (weak beam). En un futuro, la determinación precisa de la energía de falta de apilamiento exigirá el uso de la teoría elástica de medios anisótropos. Esta teoría es sumamente compleja, y exige la simulación por ordenador de la conformación de las dislocaciones en configuraciones de equilibrio precisas, tema éste que es objetivo de la colaboración hispanofrancesa que continúa con este trabajo.

\section{AGRADECIMIENTOS}

Los autores reconocen el apoyo financiero prestado por el Ministerio de Educación y Ciencia a través de la Acción Integrada Hispano-Francesa, HF2003-0272

\section{BIBLIOGRAFÍA}

1. Y. Ohmachi, and N. Uchida. Acoustic and acousto-optical properties of $\mathrm{TeO}_{2}$ single-crystal. Rev. Electr. Commun. Lab. 20, (5-6), 529, 1972.

2. Special Issue on Acousto-Optics Proc. IEEE 69, No. 11981

3. J. Janszky, A. Péter, A. Mecseki, R. Voszka, I. Tarjan, I. Földvári, M. Silvestrova, Yu V. Pisarevskii. Dislocation density and hypersound attenuation in paratellurite. Kristallografiya, 27 (1), 152, 1982.

4. V. I. Balakshy and I. M. Sinev, "Mode Competition in an acousto-optic generator", J. Opt. A: Pure Appl. Opt. 6, 469-74, 2004.

5. A. Péter, E. Fries, J. Janszky, J. Castaing. Dislocations in paratellurite $\mathrm{TeO}_{2}$ - Elastic energies and plastic-deformation. Revue Phys. Appl. 21 (5), 289298, 1986.

6. H. Ogi, M. Fukunaga and M. Hirao, "Elastic Constants, internal frictions and piezoelectric effects of $\alpha-\mathrm{TeO}_{2}$ ", Phys. Rev. B 69, 241004, 2004.

7. K. Yasutake, K. Sugiura, H. Inoue, A. Takeuchi, M. Uemura, K. Yoshii y H. Kawabe. Dislocations and ultrasonic attenuation in paratellurite. Phys. Stat. Sol. (a) 125, 489, 1991.

8. Hull, D and Bacon, D.J.. Introduction to dislocations. $3^{\text {rd }}$ edition, p. 217, 1984.

9. T. Bretheau, J. Castaing, J. Rabier and P. Veyssière; “Mouvement des dislocations et plasticité à haute température des oxydes binaires et ternaires", Adv. in Phys. Vol. 28, n6, pp. 835-1014, 1979.

10. J. Martínez-Fernández, M. Jiménez-Melendo, A. Domínguez-Rodríguez, K. P. D. Lagerlöf and A. H. Heder; "High temperature precipitation hardening of $\mathrm{Y}_{2} \mathrm{O}_{3}$ partially stabilized $\mathrm{ZrO}_{2}$ (Y-PSZ) single crystals-II. A quantitative model for the hardening", Acta metall. mater. Vol. 41, 3171-3180, 1993.

Recibido: 09.01 .05

Aceptado: 28.04 .05 